\title{
1 Title: Netome: The Molecular Characterization of Neutrophil Extracellular Traps (NETs)
}

2 Running Title: Genomics and Proteomics of NETs

4 Authors:

5 David Scieszka ${ }^{1}$, Yi-Han Lin $^{2}$, Weizhong Li $^{1}$, Saibyasachi Choudhury ${ }^{1}$, Yanbao Yu ${ }^{2}$, Marcelo 6 Freire $^{1,2,3^{*}}$

8 Affiliations

$9{ }^{1}$ Department of Human Biology and Genomic Medicine, J. Craig Venter Institute, La Jolla, CA 1092037, USA.

$11 \quad{ }^{2}$ Department of Infectious Disease, J. Craig Venter Institute, Rockville, MD 20850, USA.

$12{ }^{3}$ Department of Infectious Diseases, University of California San Diego, La Jolla, California, 13 USA.

$23 *$ Corresponding author

24 Marcelo Freire, D.D.S., Ph.D., D.Med.Sc

25 Associate Professor

26 Genomic Medicine and Infectious Diseases

27 4120, Capricorn Lane, 92037

28 La Jolla, CA

29 mfreire@jcvi.org 


\section{Abstract}

2 Neutrophils are the most abundant type of white blood cells in humans with biological roles 3 relevant to inflammation and fighting infections. The release of neutrophil extracellular DNA 4 aims to control invasion by bacteria, viruses, fungi, and tissue damage. Neutrophil Extracellular 5 Traps (NETs) act as antimicrobial agents triggering immune signaling through the release of the 6 nuclear content into the extracellular space. Although intense investigations have elucidated the 7 pathways preceding NET formation, the exact molecular composition of released NETs has not 8 been mapped. We aimed to decode the sequences of DNA and proteins from NETs. With 9 emerging needs to understand neutrophil functions precisely, we open the field of NETOMIC 10 studies through isolation of NETs in combination with omics approaches including shotgun 11 genomics and proteomics. Our in vitro NET isolation methodology allowed for unprecedented 12 replicability with induction in a sterile inflammation model system. Enrichment of mitochondrial 13 DNA and telomere sequences are significantly expressed in NET genomes. This study revealed 14 that the genomic sequence released in the extracellular milieu is not stochastically serving as a 15 scaffold for a repertoire of proteins involved in neutrophil protective functions. Collectively, we 16 established the gene and protein signatures exclusive to the extracellular NETs in comparison to 17 undifferentiated and differentiated neutrophil states, further guiding future detection of specific 18 regions needed for diagnostics and targeted therapies of NET related conditions. 


\section{Introduction}

2 As polymorphonuclear leukocytes of the phagocytic system, neutrophils are essential for early 3 immune defense in sites of infection where they kill pathogens ${ }^{1,2}$. They are generated from 4 myeloid precursors in the bone marrow prior to differentiating into several stages of maturation, 5 including myeloblast, promyelocyte, myelocyte, metamyelocyte, bands, and polymorphonuclear 6 cells. Neutrophils are the most abundant types of white blood cells in mammals - approximately 7100 billion are produced in the human body every day. From the blood circulation, neutrophils are 8 recruited by chemical and physical cues to the periphery and are able to quickly infiltrate tissues 9 and interstitial areas to detect and eliminate threats. Neutrophils present key functions in the 10 clearance of pathogens such as bacteria ${ }^{3}$, fungi ${ }^{4}$, viruses ${ }^{5}$, and parasites ${ }^{6}$. In addition to the early 11 recruitment to infection sites and clearance mechanisms by phagocytosis, oxidative burst, and 12 degranulation, another much less recognized means of cellular attack can develop. Web-like 13 chromatin structures known as neutrophil extracellular traps (NETs) are produced by neutrophils 14 and released from the intracellular regions for host protection and infection control ${ }^{7,8}$. The DNA 15 backbone of NETs is attached to molecules, such as histones, calprotectin, and cathepsin G 16 protease, which provide antimicrobial properties to eliminate invaders ${ }^{9}$. Neutrophil response and 17 NET production require a fine balance: underactivity leads to increased invasion from pathogens, 18 whereas over-activity is highly damaging to tissues. While NETs are favorable in the host defense 19 against pathogens, secondary damage to tissue from sustained formation can lead to a cascade of 20 inflammatory reactions, resulting in organ damage, cancer, tissue loss and thrombosis. When 21 dysregulated, excessive NET release has been implicated in diseases, including lupus ${ }^{10}, \mathrm{COPD}^{11}$, 22 type 2 diabetes $^{12}$, chronic inflammation ${ }^{13}$, cystic fibrosis ${ }^{14}$, autoimmunity ${ }^{15}$, and cancers ${ }^{16}$, among 23 others ${ }^{17,18}$.

24 Accumulating evidence supports the notion that expelling DNA to the extracellular environment 25 is not generally considered a specialized immune function, it was thought that the release was not regulated, and that molecules involved were randomly placed in the extracellular environments. It 27 is now accepted that this process is a fine-tuned and well-controlled intracellular process. Known 28 factors guiding generation of NETs include neutrophil elastase (NE), peptidyl arginine deiminase 29 type 4 (PADI4), and gasdermin D. The release is dependent on the type, concentration, and 30 duration of stimulus presented ${ }^{1,4,8,16,18,19}$. Stimuli from microbes and chemicals act differently in 31 activation, cell membrane rupture, and NET expulsion. In response to a more replicable 32 substance, such as phorbol 12-myristate 13-acetate (PMA), common intracellular pathways are 33 activated in neutrophils, including protein kinase $\mathrm{C}(\mathrm{PKC})$-mediated pathways, and MAPK/ERK 
1 signaling which generates downstream reactive oxygen species (ROS) via NADPH oxidase and 2 myeloperoxidase (MPO). In fact, MPO is believed to have several roles in NET formation 3 pathways upon its release from azurophilic granules. Once activated, MPO can convert $\mathrm{H}_{2} \mathrm{O}_{2}$ into 4 hypochlorous acid ( $\mathrm{HOCl}$ ), which is one of the most caustic chemicals used in NETs to terminate 5 invading pathogens. MPO also utilizes ROS to mediate the activation of NE which translocates 6 into the nucleus for initial histone degradation and chromatin unpacking. MPO further promotes 7 chromatin unpacking by activating PADI4, which is responsible for the citrullination of histones.

8 However, most of the scientific evidence was focused on the identification of intracellular 9 pathways leading to NET release and not on dissecting the content expelled from neutrophils.

10 There are multiple molecules released from neutrophils that are unknown, highlighting the need 11 to decode the exact sequences of the human NETs.

12 Despite emerging interests in neutrophil phenotypes, their use in research experiments are limited 13 due to the short lifespan of the cells, high sensitivity to handling and temperature, making it 14 challenging to achieve replicability. To revisit these issues and determine NET composition, we 15 devised a protocol that reliably produced NETs in a sterile inflammation setting. We leveraged a 16 myeloid undifferentiated cell line (HL60 cell line) into our cell differentiations systems (dHL60). 17 Through concentration-depended and time point PMA perturbation studies, we successfully 18 transformed neutrophils into NET producing cells. Here, we aimed to identify the genomic 19 sequence of NETs and to characterize their molecular contents (specific proteins, and metabolic 20 markers for NET scaffold). We demonstrated through imaging, staining, sequencing, and bacterial 21 clearance that the sequences were viable NET materials. The NET sequences were compared to 22 undifferentiated neutrophils and differentiated neutrophils. Finally, we compared our data with a 23 published dataset to understand the replicability of our model to other neutrophil models. Our

24 study showed a replicable system to produce human NETs and identified the genomic and 25 proteomic contents of the differentiated neutrophils. This first human sequence study determining 26 the exact content material opens new avenues for elucidating NETs structures.

\section{Materials and Methods}

\section{Experimental Design}

29 To achieve our goal of creating an in vitro NET pipeline, promyeloblast human cell line HL-60 30 cells were selected due to their ability to differentiate into neutrophils. Kinetic studies were 31 performed to validate the timeline of differentiation and different concentrations of PMA that 
1 were required to induce optimal NET release. These were validated through visual inspection,

2 immunofluorescence, MitoSOX-red assays, NanoDrop, and Qubit.

4 Promyeloblast human cell line, HL-60, was acquired from ATCC. HL-60 cell lines were 5 maintained in culture media prepared according to manufacturer's guidelines. Briefly, the HL-60 6 culture media consisted of Iscove's modified Dulbecco's medium (IMDM, Gibco, cat. 7 no. 12440-061) with 5\% fetal bovine serum (FBS, heat inactivated, Gibco, cat. no. 26140-095) 8 and 1X antibiotic (Penicillin + Streptomycin; Gibco, cat. no. 15-140-122). Note: Avoid using 9 antimycotic in the recovery media as it affects the recovery and growth of HL-60 cell lines.

\section{2- Differentiation of HL-60 to Neutrophil (dHL-60)}

11 HL-60 cells in culture media were centrifuged at $275 \mathrm{x}$ g for 10 minutes at room temperature (RT) 12 and the culture media was aspirated. Cell pellets were resuspended in differentiation media (i.e 13 culture media containing $1.5 \%$ dimethyl sulfoxide) to an initial cell seed count of 10E5 cells. 14 Verification of a previous neutrophil differentiation timeline was performed ${ }^{20}$. To quantitatively 15 determine the maximum number of differentiated cells before apoptosis, a growth curve was 16 created using a 96-well plate measured daily for 5 days. Morphological changes were monitored 17 by Giemsa staining through the comparison of HL-60 cells to differentiated HL-60 cells (dHL-60) 18 i.e. neutrophils.

\section{3-Neutrophil Extracellular Trap Production}

20 Multiwell plates were used in kinetic studies to determine effective NET release from dHL-60 21 cells. PMA was used to induce NET release at concentrations ranging from $0.1 \mathrm{nM}-8,000 \mathrm{nM}$ 22 and at time points ranging from 10 minutes to 6 hours. Morphological changes were monitored 23 through different microscopy techniques and DNA was quantified by NanoDrop 24 spectrophotometer, Agilent High-sensitivity DNA chip using Bioanalyzer 2100 (Fig. 1E), and 25 QuBit 2.0.

\section{4-DNA Isolation from $\mathrm{HL}-60, \mathrm{dHL}-60$, and NET}

27 HL-60 and neutrophil (dHL-60) DNA was isolated via the Qiagen AllPrep DNA/RNA/Protein 28 Mini Kit according to the manufacturer's protocol ${ }^{21}$ and suspended in an elution buffer (EB) for 
1 storage at $-20^{\circ} \mathrm{C}$. DNA purity was quantified with NanoDrop spectrophotometer, QuBit 2.0, and 2 Agilent High-sensitivity DNA chip.

3 We adopted a previous protocol for NET production from primary blood cells ${ }^{22}$. After 4 hours of 4 incubation in PMA, culture media was aspirated to remove non-NET forming cells. Adherent, 5 NET-releasing cells were resuspended in ice-cold PBS(-) to a final volume of $12 \mathrm{~mL}$. The 6 solution was centrifuged at $275 \mathrm{x}$ g for 10 minutes to pellet cells. The NET-rich supernatant was 7 transferred to an ultracentrifuge tube and the cell pellet was discarded. Ultracentrifugation was 8 performed at $18,000 \times \mathrm{g}$ for 10 minutes at RT to pellet DNA and the PBS(-) supernatant was 9 aspirated. NET DNA pellets were resuspended in EB for storage at $-20^{\circ} \mathrm{C}$.

\section{$10 \quad$ 5-MitoSOX staining and Flow Cytometry}

11 The On-Chip Sort flow cytometer was used to quantify neutrophil ROS generation by staining 12 with MitoSOX. Multiwell plates were used to compare HL-60 and dHL-60 cells in PBS(-) and in 13 PMA over the course of 5 hours at 30-minute increments. In total, three experimental replicates 14 were performed and analyzed.

\section{$15 \quad$ 6-Cell Staining and Microscopy}

16 To monitor nuclear morphological changes, HL-60 and dHL-60 cells were stained with Eosin Y, 17 Hematoxylin and Giemsa according to conventional methods and viewed under a histological 18 microscope. Fluorescent staining was visualized using a Leica TCS SP5 II confocal microscope to 19 qualify NET DNA release when compared to E. coli co-incubation. Both groups were 20 permeabilized, stained with DAPI (1,000 nM), fixed with 4\% PFA for 15 minutes, and checked 21 for fluorescence. Additionally, nuclear morphology changes and NET release were visualized via 22 3D-Cell Explorer Nanolive microscope under fixed, and unfixed conditions. Comparative 23 visualizations of isolated HL-60, dHL-60, and NET DNA was further performed using scanning 24 electron microscopy.

\section{$25 \quad$ 7-Scanning Electron Microscopy}

26 HL-60, neutrophil, and NET DNA samples were retrieved from $-20^{\circ} \mathrm{C}$ and allowed to thaw on 27 ice. Once thawed, samples were dehydrated in ethanol, embedded in epoxy resin, sectioned at 50$2860 \mathrm{~nm}$ on a Leica UCT ultramicrotome, and picked up on Formvar and carbon-coated copper 29 grids. Sections were stained with 2\% uranyl acetate for $5 \mathrm{~min}$ and Sato's lead stain for $1 \mathrm{~min}$. 
1 Grids were viewed using a Tecnai G2 Spirit BioTWIN transmission electron microscope

2 equipped with an Eagle 4k HS digital camera (FEI).

3 Formvar-carbon-coated copper grids (100 mesh, Electron Microscopy Sciences, Hatfield, PA) 4 were placed on $20 \mu 1$ drops of each sample solution displayed on a Parafilm sheet. After allowing 5 material to adhere to the grids for 10 minutes, grids were washed 3 times by rinsing through 200 $6 \mu \mathrm{l}$ drops of milli-Q water before being left for $1 \mathrm{~min}$ on $2 \%$ (wt/vol) uranyl acetate (Ladd 7 Research Industries, Williston, VT). Excess solution was removed with Whatman 3M blotting 8 paper, and grids were left to dry for a few minutes before viewing. Grids were examined using a 9 JEOL JEM-1400Plus transmission electron microscope operating at $80 \mathrm{kV}$. Images were recorded 10 using a Gatan OneView 4K digital camera.

\section{8-Proteomics}

12 Isolation of HL-60 and dHL-60 protein was conducted via the Qiagen AllPrep DNA/RNA/Protein 13 Mini Kit and conducted according to the manufacturer's instructions ${ }^{21}$. Attempts using the 14 AllPrep to isolate NET protein from total NET samples were unsuccessful and the total NET 15 isolation protocol was used for proteomics analysis.

\section{9-NET protein preparation for proteomics}

17 After thaw from $-80^{\circ} \mathrm{C}$, NET solutions were added with protease inhibitors and $1 \%$ Benzonase 18 Nuclease (Sigma) and incubated at $37^{\circ} \mathrm{C}$ for $20 \mathrm{~min}$ to remove nucleic acids. Protein samples 19 were then processed using the Suspension Trapping (STrap) approach as described previously ${ }^{23}$ to 20 generate tryptic peptides for liquid chromatography-tandem mass spectrometry (LC-MS/MS) 21 analysis. To specifically identify nucleic acid-bound proteins, one set of NET samples were 22 processed similarly but excluded the Benzonase treatment.

23 In total, LC-MS/MS was performed on 6 protein samples (three NET with Benzonase treatment 24 and three NET without Benzonase treatment) following a protocol described previously ${ }^{24}$. In 25 brief, the desalted samples were first resuspended into $20 \mu 10.1 \%$ formic acid in water and then 26 loaded onto a trap column $(2 \mathrm{~cm} \times 300 \mu \mathrm{m}$, PepMap C18, Thermo Scientific $)$ and an analytical 27 column $(19 \mathrm{~cm} \times 75 \mu \mathrm{m}, 3.0 \mu \mathrm{m}$; ReproSil-Pur C18-AQ media) coupled to an Ultimate 3000 28 nano-LC and Q-Exactive mass spectrometer system (Thermo Scientific). Protein identification 29 and quantitation were performed using Proteome Discoverer (version 2.2) and MaxQuant-Perseus 30 software suite. The UniProt human database (20,413 sequences, reviewed only; version 2018_12) 
1 was used for protein search. Only the peptide and protein identifications with false discovery rate

2 (FDR) of $1 \%$ or less were accepted in the final data set. More details of proteomic procedures can

3 be found in the recent publication ${ }^{24}$. The mass spectrometry proteomics data have been deposited

4 to the ProteomeXchange Consortium via the PRIDE partner repository with the dataset identifier

5 PXD016143.

\section{0-Genomic Sequencing}

7 Illumina's NexteraXT library prep kit was used for HL-60, dHL-60, and NET samples and 8 sequenced on the NovaSeq 6000 platform using an S2 flow cell 2X150bp. 400 pM of each sample 9 pool was loaded and 1\% PhiX was spiked in each lane ${ }^{25}$. Cluster density was $2961 \mathrm{~K} / \mathrm{mm} 2$ with $1080 \%$ PF. $1429.42 \mathrm{~Gb}$ and 4.5B PE Reads were generated. Coverage for the HL-60, dHL-60, and 11 NET samples were $36 \mathrm{X}, 45 \mathrm{X}$, and 47X respectively. The raw genomic sequences are available at 12 NCBI Sequence Read Archive (SRA) under BioProject under accession PRJNA587717 at 13 https://www.ncbi.nlm.nih.gov/bioproject/PRJNA587717.

14 The resultant fastq files were input into the CLC Workbench (v11), trimmed by $15 \mathrm{nt}$ on the 3' to 15 remove primers, trimmed for q-scores $<25$, mapped to the human genome (hg38, CLC v12), and 16 checked for normal human GC content. To determine genomic enrichment, a sliding window 17 analysis was conducted for every 20, 100, 500, and 5,000 nt. Regions of interest (ROIs) were 18 selected based on known protein association", proteomics results, and nonselective general 19 analysis. Sliding window files of aforementioned sizes are available through the corresponding 20 author. Additionally, unmappable read files are also available and were not used in our analysis.

21 Normalization Steps for Genomics Sliding Window

22 Normalization of sliding window coverage was performed by dividing coverage per sliding 23 window by complete coverage per sample per chromosome.

\section{Eg. 5,000bp Window Coverage for NET on Chromosome 1 $\div$ Total Sample Coverage for NET on Chromosome 1}

25 Normalization of mitochondrial DNA was performed by dividing coverage per sliding window by 26 complete coverage per sample for the entire genome. 


\section{Eg.20bp Window Coverage for NET on Chromosome MT $\div$ Total Sample Coverage for NET Chromosomes}

2 Known Protein Association

3 Proteins known to be localized within NETs were tested for genomic enrichments or depletions

4 based on normalized 20 nt sliding windows ${ }^{9}$. These proteins of interest (POIs) were input into

5 Ensembl biomart (Release 96) and their genomic start/end locations were determined.

\section{Proteomic Protein and RNA Expression Selection}

7 Proteomics analysis was used to retroactively determine ROIs. The genomic regions associated 8 with the protein results were input into Ensembl biomart and their genomic start/end locations 9 were determined. Enrichment/depletion analysis was performed with 20 nt normalized sliding 10 windows.

\section{Nonselective General Analysis used for RNA Expression Overlap}

12 Using the $5000 \mathrm{nt}$ normalized window analysis, regions were selected based on 1.5-fold-change 13 for each sample compared to the others simultaneously or compared to the average. The annotated 14 list used for exonic analysis was acquired from Ensembl biomart and used in conjunction with the 15 normalized 5,000 nt windows. Overlapping expression analysis was performed by downloading 16 the neutrophil gene expression data table 17 (http://collinslab.ucdavis.edu/neutrophilgeneexpression/), and comparing the gene list generated 18 from our nonselective general analysis. The initial range of genomic coverage spanned several 19 orders of magnitude and a percentage of the total became more representative for visualization. 20 Namely, each sample was divided by the average of all three samples.

\section{Eg.NET GeneX Coverage $\div$ [(NET GeneX + HL60 GeneX + dHL60 GeneX $) / 3]$}

22 It should be noted that this normalization was simply for aesthetic reasons (Fig. 3) and was not 23 performed during our initial genome-wide scan. All circos plots were generated using the 24 ShinyCircos software (http://shinycircos.ncpgr.cn/).

25 Neutrophil elastase fluorescence and growth curves were monitored using the Celigo $\mathrm{S}$ cell 26 imaging cytometer. Briefly, coculture experiments with Fusobacterium were performed in 6-well 
1 plates at a 1:10 ratio of neutrophils to bacteria, respectively. Standard curves were generated 2 according to manufacturer instructions. Celigo internal cell counting software was utilized to 3 determine cell growth each day over 5 days to determine cell division and terminal differentiation 4 in 6-well plates.

5 Statistical significance was calculated with a nonparametric Mann-Whitney test and Prism 6 software (Graphpad Software Inc.). Statistical significance for proteomic dataset was calculated 7 with ANOVA. Genomic statistical significance for NETs, telomeric frequency, and mitochondrial 8 enrichments were calculated using ANOVA. Statistical significance for correlations was 9 calculated by Spearman rank test with $\mathrm{P}$ values and $\mathrm{r}$ values noted on the respective graphs. $10 * \mathrm{P}<0.05, * * \mathrm{P}<0.01, * * * \mathrm{P}<0.001$, and $* * * * \mathrm{P}<0.0001$ were considered significant and are referred 11 to as such in the text.

\section{Results}

\section{Neutrophil Differentiation and NET Release Model}

14 To develop a neutrophil differentiation protocol and produce NETs in vitro, we first established a 15 differentiation system that allowed cells to be viable with synchronized NET production. In 16 mimicking human neutrophil differentiation, cells need to be primed prior to a specific response 17 such as oxidative burst, phagocytosis or $\mathrm{NET}^{26}$. Extensive literature related to HL60 cells have 18 made this an attractive model for studies of differentiation. Because neutrophil differentiation 19 protocols lead to increased cell death, we established a threshold of differentiation that would 20 keep viability at 80\%. HL60 cells grow in suspension culture with a doubling time that can vary 21 from 20-45 hrs. Morphologically, the cells present with rounded nuclei and basophilic cytoplasm 22 with azurophilic granules (Figs. 1B, S1A). The majority of the cells carry a variety of 23 histochemical markers characteristic of myeloid lineage, most notably myeloperoxidase and acid 24 phosphatase. We evaluated to confirm cell morphology histochemically through trypan blue, 25 Giemsa, and by flow cytometry analysis (CD11b). We initiated testing cell differentiation by 26 culturing undifferentiated HL60 with culture medium enriched for DMSO (0.1-10\%). As 27 compared to steady state, neutrophil differentiation lead to cytoplasm enlargement, loss of 28 primary granules, emergence of secondary granules, nuclear condensation and segmentation. We 29 then established neutrophil differentiation at the viability threshold, with morphological 30 characteristics and surface marker validation at 5 days under 1.5\% DMSO (Figs. 1B, S1A). As 
1 expected, cells were terminally differentiated and presented phenotypic characteristics of

2 molecular neutrophils.

3 One of the key characteristics to discriminate neutrophil differentiation to monocyte lineage from 4 the progenitor cells is their nuclei shape and production of classic neutrophil enzymes such as 5 elastase, myeloperoxidase, and ROS formation. We therefore further confirmed that our cells 6 followed the fate of mature neutrophils rather than macrophages. Once HL-60 cells were 7 differentiated into mature neutrophils, we performed kinetic studies to investigate NET release. 8 Our pilot experiments with live imaging cytometry assayed the optimal concentrations of PMA 9 stimulation $(1-10,000 \mathrm{nM})$. Previous data indicated that higher and lower concentrations under 10 longer periods of time were able to induce $\mathrm{NETs}^{27}$. In our system we achieve synchronized NET 11 formation by applying PMA $(1,000 \mathrm{nM})$. In the same experiment we investigated the kinetics to 12 understand the ideal time for NET release (0-6 hours). Our morphological analysis demonstrated 13 that at 4 hours cells were viable with morphological changes leading to spike NET formation. It is 14 known that prior to NET release, mitochondrial superoxide is formed along with reactive oxygen 15 species. We evaluated our cells by either morphological analysis or MitoSOX-red staining of 16 mitochondrial superoxide (Figs. 1B, D, S1A, B). At 2.5 hours, our results showed a peak of ROS 17 formation leading to increased staining of MitoSOX (Figs. 1D, S1B). Comparatively, neutrophils 18 in PMA were $75.23 \%$ positive for MitoSOX versus $14.98 \%$ in PBS while HL60 cells in PMA 19 showed $23.05 \%$ positive versus $10.89 \%$ in PBS. These early events preceded NET formation and 20 were replicable through flow cytometry monitoring. Quantitatively, stimulation increased the 21 number of oxygen species which allowed the separation of cells into three groups: MitoSOX ${ }^{\text {lo }}$, 22 MitoSOX $^{\mathrm{Mid}}$, MitoSOX ${ }^{\text {high }}$, with intermediary subsets of MitoSOX ${ }^{\text {high }}$ representing the producers 23 of high amounts of NETs.

24 The phenotypic identity of neutrophils was further confirmed through viability staining (SYTOX 25 and DAPI). While the expression levels of neutrophil proteins were not affected by the high PMA $1,000 \mathrm{nM}$ concentration, the viability decreased to $50 \%$ at 5 hours and $30 \%$ at 6 hours. It is possible that to produce NETs from leukemia cell derived cells, the genetic profiling is different when compared to primary cells which have low viability in ex vivo settings. Upon selection criteria determination for NET induction and release (4 hours and 1,000 nM) of differentiated 30 cells, we proceeded to isolate NETs by adapting a protocol previously described ${ }^{28}$. We 31 confirmed that a minimum neutrophil count of 1.0E6 was needed for measurable DNA isolation.

32 We next confirmed the expression of DNA release by nucleotide staining, gel analysis, 33 spectrophotometry and scanning electron microscopy (SEM). We controlled our assays by 
1 comparing the NET isolated groups with the undifferentiated cells and differentiated cells.

2 Clearly, the NET groups present morphology and readings to DNA. To ensure our NET materials

3 presented with isolated DNA from the extracellular sites and not from the cells, we visualized the

4 isolated materials through SEM. The only group demonstrating thread-like structures and web-

5 like structures was the NET group from the isolation layer - acquired from NETs adherent to the

6 culture flask after PBS wash - while other phase layers of the high centrifugation protocol failed

7 to demonstrate these patterns. To confirm the molecular content, DNase was applied to the

8 isolated samples and compared to DNase free isolates (Fig. 1E). When we applied DNase to the

9 material released, we showed that quantitatively and through imaging that the structures and DNA

10 diminished, further confirming the nucleotide nature of NETs. The presence of lipid bilayers in

11 SEM images of NETs could also be vesicles, which are commonly known to be released by

12 neutrophils (Fig. 1C). Collectively we defined a method to differentiate neutrophils, and to

13 produce and isolate NETs. Following our validation protocols, we proceeded to extract

14 DNA/RNA/protein from our three groups for proteomics and genomics sequencing.

\section{NET Proteome Analyses}

16 The analysis of proteomics data revealed significant differences on the NET groups compared to

17 undifferentiated and differentiated neutrophils in expression levels of proteins known to be 18 associated with neutrophil function and NETs. To ensure the protein content was from NET and 19 not from cells, our system allowed us to investigate proteins that were exclusive to NET groups 20 and not the cell groups. Principal component analysis showed that NETs presented unique 21 clusters when compared to cell proteins (Fig. S4). Out of initial 2,403 proteins quantified in 22 NETs, 316 proteins were enriched when compared to neutrophils and undifferentiated cells 23 (HL60; Fig. S5). Although the isolated NETs presented with unique and overlapping sequencings, 24 this group was the only one to demonstrate enrichments for classic pathways for NET formation, 25 confirming our DNA and cytometry findings. In line with the specific protein signatures, 26 differentiated and undifferentiated cells showed reduced numbers of NET markers and presented 27 the highest overlapping rates with pathways related to extracellular exosomes, mitochondrial 28 metabolism, mitochondrial nucleoid, and extracellular matrices (Fig. S5).

29 We next assessed isolated samples from NET groups and cells were processed for shotgun 30 proteomic analyses to characterize the protein identity and function. One set of samples were 31 directly processed using the established method to generate tryptic peptides for LC-MS/MS 32 analysis $^{24}$, while the other set of samples were treated with Benzonase to degrade all DNA and 
1 RNA contents prior to tryptic digestion. Because Benzonase is able to cleave the condensed 2 nucleic acid component in collected NET, we monitored its activity to subsequently release 3 proteins that are tightly bound to the nucleic acids, thus enhancing proteomic mapping ${ }^{29}$. Without 4 Benzonase treatment, the abundance of protein levels was moderate with 1,711 proteins 5 identified. Whereas after Benzonase treatment an increased abundance of proteins was found, 6 with identification of 2,364 proteins (Fig. 2A). The proteome size spans higher than five orders of 7 magnitude, and in addition to novel protein findings, the protein functions previously associated 8 with NET in comparison to our study findings is listed (Fig. 2B) ${ }^{9}$, further validating the 9 enrichment of NETs in our culture and isolation system.

10 Functionally, the 100 most abundant proteins identified in our proteome analysis included histone 11 proteins $\mathrm{H} 4, \mathrm{H} 2 \mathrm{AFV}, \mathrm{H} 2 \mathrm{AJ}$, and $\mathrm{H} 3 \mathrm{~F} 3 \mathrm{~A}$, which are known to be the core components of 12 nucleosome and play a central role in cell functions including transcription regulation, 13 chromosome stability, DNA repair and DNA replication. Consistently, a classic NET enzyme, 14 Myeloperoxidase (MPO), which was evaluated by flow cytometry in our culture experiments was 15 also found in high abundance. This was also evident for the calcium and zinc-binding proteins 16 known to be released by blood neutrophils in humans after migrating to the site of inflammation, 17 calprotectin (S100A8 and S100A9), which plays an important role in the regulation of 18 inflammation and immune response ${ }^{30}$.

19 The Label Free Quantitation (LFQ) intensities correlate well within the same treatment groups 20 (Fig. S6), showing consistent reproducibility of NET proteome profile. Overall, 2,280 proteins 21 were common within the NET groups, and 126 proteins have more than two-fold difference 22 between the two groups by statistical analysis (Permutation FDR 0.05, Fig. 2C). Histone proteins, 23 cathepsin G, S100A8, S100A9, and azurocidin have more than two-fold increase in the 24 Benzonase-treated group, indicating their tight association with the condensed nucleic acid 25 component in NET. Upon functional analysis of the 101 proteins with at least two-fold increase in 26 the Benzonase-treated group, we found that while a fraction of the proteins is involved in 27 leukocyte activation and neutrophil degranulation $(\mathrm{FDR}=7.15 \mathrm{E}-5)$, another group of proteins 28 related to RNA binding $(\mathrm{FDR}=0.0143)$ was also enriched (Fig. 2D). We attempted to extract 29 RNA from NETs, but were not able to isolate detectable levels. However, the proteomic findings 30 indicate that the condensed nucleic acid component from NET may not only be the chromosome 31 scaffold, but also includes cellular RNAs. 
1 Given the abundance of novel proteins, we sought out alternative data organization methods for

2 pathway enrichment analyses to test against our subsequent genomic sequencing. As such, we

3 organized the proteomic score by peptide spectrum matches (PSM) and input the resultant protein

4 hierarchy into the panther gene ontology pipeline (http://www.pantherdb.org/), which

5 demonstrated pathways associated with positive regulation of neutrophil degranulation, cytosol,

6 mitochondrial proteins and RNA binding molecules. In addition to the abundance of

7 mitochondrial proteins, NETs contained a statistically significant increase in the telomere

8 sequence TTAGGG from our genomics sequencing (Fig. 4B). To our knowledge, this is the first

9 evidence that neutrophils could be increasing the amount of DNA to be released in the NET

10 process via the extension of telomeres through the internal RNA template of human telomerase

11 reverse transcriptase.

\section{NET Genome Analysis}

13 Sliding Window Analysis for Non-Targeted Genome-Wide Scan

14 To interrogate the genomic sequence of the scaffold DNA released by neutrophils, we utilized 15 Illumina's NexteraXT library prep kit and sequenced NET genome on the NovaSeq 6000 16 platform. Coverage for isolated Nets were compared to undifferentiated cells and differentiated 17 cells $(36 \mathrm{X}, 45 \mathrm{X}$, and $47 \mathrm{X}$ respectively). The raw genomic sequences were submitted to NCBI 18 Sequence Read Archive (SRA) under BioProject under accession PRJNA587717 at 19 https://www.ncbi.nlm.nih.gov/bioproject/PRJNA587717.

20 The resultant fastq files were input into the CLC Workbench (v11) software, trimmed by $15 \mathrm{nt}$ on 21 the 3' end to remove primers, trimmed for q-scores < 25, mapped to the human genome (hg38, 22 CLC v12), checked for normal human GC content, and comparatively scanned using a sliding 23 window analysis ${ }^{31}$ which was normalized to each sample's chromosome. To determine genomic 24 enrichment, a sliding window analysis was conducted for every 20, 100, 500, and 5,000 nt, with 25 the $500 \mathrm{nt}$ represented for mitochondrial DNA (Fig. 4D) and the 5,000 nt results represented for 26 whole genome analysis (Figs. 3, S2). Regions of interest (ROIs) were selected based on known 27 functions $^{9}$, proteomics results, and nonselective general analysis. Unmappable read files were not 28 used in our analysis. From the sliding window approach, we were able to reveal regions of 29 enrichment and depletion within each sample.

30 After the genome-wide scan was conducted, comparative analyses were made for each possible 31 grouping of samples using a 1.5-fold enrichment/depletion cutoff. From this, each sample 
1 grouping revealed many intronic and exomic regions of interest. Most notably, there were 25,488

2 regions in the NET when compared to the HL-60 and neutrophil sample groups (Fig. S3F),

3 whereas HL-60 showed this in 6,147 regions (Fig. S3D) and neutrophil showed this in 5,351

4 regions (Fig. S3E) Overall, there were 538,355 regions within this analysis that showed no

5 consistent enrichment or depletion when compared to any possible grouping (Fig S3G). We

6 retrieved the genes associated with the 25,488 regions and represented them in figure S2. These

7 data could suggest that NET DNA scaffold is being released in a time-dependent manner due to

8 the regions of NET enrichment and depletion being 4-fold higher than neutrophil or HL-60, with

9 HL-60 and neutrophil regions being relatively similar to each other.

10 We reasoned whether DNA was being released in an ordered fashion, i.e. DNA not deemed 11 essential for immediate survival or NET release was being preferentially ejected first. To 12 rationalize the sequence of NETs with neutrophil function, we looked into known RNA-seq 13 datasets related to similar cell lineage. In the case of NET release, the expression of survival 14 genes would necessarily require them to be inside of the neutrophil as other DNA is being ejected. 15 To ensure our sequences align, we probed previously published data HL-60 undifferentiated and 16 differentiated RNA-seq data deposited at the NCBI Gene Expression Omnibus (GEO) database 17 (Accession: GSE103706) along with previously published data for primary human neutrophils 18 from the Wright et al [GEO: GSE40548], Jiang et al [GEO: GSE66895]. This comprehensive list 19 was compared to our NET enrichment/depletion list. Our findings demonstrated that out of a list 20 of 37,470 RNAs, 400 overlapping regions were determined (Fig. 3), indicating enrichments and 21 depletions of NET genes present in the extruded materials.

\section{Sliding Window Analysis for Targeted Candidate Gene Regions}

23 We next sought to ascertain what DNA sequences were enriched in NET samples when compared 24 to the neutrophil groups. We performed an additional targeted candidate analysis of genes related 25 to the known NET release pathway(s). Previous studies of genes known to be implicated in NET 26 release showed statistically significant DNA depletion in our NET samples, such as PADI4 and 27 MPO ( $\mathrm{p}<0.05$, Fig. 4A). We investigated genomic telomere enrichments using targeted window 28 analysis (TTAGGGTTAGGG). Telomere counts in NET sequences showed statistically 29 significant increase over undifferentiated and differentiated neutrophil telomere counts, while HL3060 and neutrophil telomere counts were not significantly different from each other $(\mathrm{p}<0.05$, Fig. 31 4B). We further identified that mitochondrial sequences showed significant enrichment in NETS 32 when compared to HL60 and neutrophil counts, whereas HL-60 and neutrophil mitochondrial 
1 enrichments were not significantly different from each other $(\mathrm{p}<0.05$, Fig. 4C). After a positional mapping comparison, the mitochondrial regions of specific NET enrichment were determined to

3 be the NADH dehydrogenase subunit components ND1, ND2, ND4, and ND5 (Fig 4D). These

4 data suggest that PMA-induced NET release is comprised of mitochondrial and nuclear DNA and

5 that neutrophils could be upregulating telomerase in order to maximize the amount of DNA being

6 released into the extracellular space. Taken together, these could suggest an ordered release

7 mechanism that preferentially ejects DNA not deemed necessary for the NET release process

8 before complete DNA ejection takes place.

9 To validate that our NETs were functional and presenting antimicrobial properties, we incubated 10 with a pathogenic strain of gram-negative microbe (Fusobacterium) and investigated the 11 microbial viability and the rate of NETs via elastase. As observed in ex vivo and in vivo settings ${ }^{32}$, 12 NETs were detectable earlier than the 4 hours required by PMA. Through microscopy, we 13 monitored NET formation timepoints and observed an increase of NET formation through a 14 survey of markers for NET quantification. Expression of neutrophil elastase was increased with $F$. 15 polymorphum over neutrophils in PMA, while neutrophils in PMA had increased expression over 16 our negative control (neutrophils in PBS), with the other strains of F. nucleatum and F. animalis 17 having no difference when compared to neutrophils in PMA (Fig. S1C). Thus, NET formed ex 18 vivo, augmented with specific bacterial strains facilitate inflammation activation and bacterial 19 clearance over 4 hours.

\section{Discussion}

21 An excessive and persistent neutrophil response is involved in the pathology of an array of human 22 conditions, and NETs have consequently become an attractive therapeutic target. Yet, therapeutic 23 strategies looking into improving pathogenic inflammation have failed to fully consider the 24 potential role of DNA sequences released by these cells. Mature neutrophil differentiation is often 25 associated with increased cell death and NET release. We set out to determine the protocol in 26 vitro that mostly produced NETs with replicability. We compared most commonly used 27 differentiation agents including DMSO, PMA, LPS, formaldehyde (Fig. S1A), selected DMSO 28 for differentiation, and tested the NET release in dose-response and time-dependence (0-6 hours) 29 through microscopy and by early (MytoSOX) and late differentiation markers (CD11b). By 30 monitoring their ability to differentiate from HL60 to dHL60 we were able to characterize their 31 morphology precisely along with decrease in cell size, increased nuclear pyknosis, and 32 segmentation along with marker expression (Figs. 1, S1A). This in vitro differentiation made the 
1 HL-60 cells ready for NET formation at 4 hours in 1,000 nM PMA. Throughout the course of our

2 NET studies, we also had the opportunity to visualize an unparalleled amount of neutrophil NET

3 formations in response to sterile inflammation and co-culturing experiments with different strains

4 of bacteria (Fig. S1C). From these, several trends emerged, such as the seemingly directed NET

5 release towards bacterial colonies when present, and NET release perpendicular to culture media

6 flow in the presence of PMA.

7 To further assess the degree of differentiation of the cells, we assessed nuclear size and 8 morphology through live imaging in a holotomography model. The cells were further isolated and 9 processed for proteomics. Although further studies are needed to confirm the molecular 10 composition of sterile PMA-induced NET release, our initial proteomic results confirm the 11 existence of known NET-associated proteins. The origin of NET DNA remains controversial, 12 with studies reporting nuclear, mitochondrial, or both depending on the cell type and stimulus ${ }^{33-}$ $13{ }^{36}$. Here, the existence of mitochondrial enrichment as well as nuclear DNA adds to the body of 14 evidence showing PMA-induced NET release has origins in the nucleus as well as mitochondria. 15 Protein comparisons of NETs to cells in both proteomics (Figs. S4, S5) and genomics (Fig. S3) 16 demonstrated that mitochondrial metabolism was highly enriched.

17 The proteomic studies, specifically, demonstrated that NETs are associated with a significant 18 amount of proteins. The greater amount of proteins identified from Benzonase-treated NETs 19 versus the untreated ones $(2,364$ vs 1,711$)$ indicates that many proteins interact tightly with the 20 nucleic acids while being released from the neutrophils. Proteins significantly increased after 21 Benzonase treatment are enriched in those involved in neutrophil degranulation, nucleus and 22 mitochondrial proteins, and membrane proteins (Fig. 2D). Although not investigated in this paper, 23 it is interesting to ponder whether Cdc42 and GPCRs could be involved in a directional NET 24 release depending on environmental cues such as shear forces or chemoattractants. NET 25 formation pathways are still under investigation, and the complexity of understanding these 26 processes relate to the fact that neutrophils are short-lived cells and not amenable to gene editing. 27 Alternatively, strategies that seek to promote neutrophil survival and to modulate NET release to 28 reduce inflammation, but simultaneously activate a more robust innate immune response, will 29 leverage the understanding of NET sequences in the extracellular niche. Emerging pathways will 30 be found from replicable approaches such as those utilized in this report. Thus, in addition to 31 revealing the number and type of proteins attached to the NETs we have also categorized them 32 according to common neutrophil functions. 
1 Our initial study of the molecular content within NETs has opened many questions, including 2 cellular processes that increase the amount of DNA being released through telomeric expansion as 3 well as region-specific NET release for prolonging cellular responsiveness. With the 4 understanding that there are at least two types of NET release - vital and suicidal ${ }^{37}$ - we reasoned 5 that the neutrophils could have secondary internal recognition pathways which could 6 preferentially sequester genomic regions for maximal longevity. PMA typically involves suicidal 7 NET release. However, the sequestration of genomic scaffolding could be time dependent, 8 meaning that certain regions could be consistently released before others. Our findings of specific 9 NET release-associated genomic scaffold depletions (Fig. 4) could imply this to be true. Further 10 exploration utilizing super resolution microscopy and DNA painting could elucidate these 11 mechanisms with greater resolution. Additionally, the finding of significant telomere enrichment 12 within the NET samples was surprising, but an interesting and unexplored avenue. In conjunction 13 with the DNA sequestration rationale, we reasoned that neutrophils could be increasing the 14 internal stockpile of DNA before ejection toward invading pathogens. From the perspective of 15 biological load, this would allow for maximum efficiency of pathogen binding and 16 immobilization at a lower cost relative to the complete elimination of a second neutrophil 17 undergoing NET release. Additional studies are needed to survey the specificity and diversity of 18 NETs in the context of their niches and cell types, while taking into consideration differentiation 19 states, health, and disease.

20 Finally, we wanted to compare our results obtained with the dHL60 cells induced NET formation 21 to a database of neutrophils ${ }^{38}$. This validation showed that our protocol had optimal 22 differentiation by using PMA. When analyzing the global gene expression in myeloid cell lines a 23 total of 400 genes were similar to RNA-seq data from undifferentiated and differentiated cells. To 24 account for measurement noise, we repeated our sequencing experiment in three independent 25 samples. For comparison, we collated raw datasets for our cell lines with the dataset from 26 previously published studies and analyzed these data sets along-side our own using identical 27 parameters and normalization strategies (Fig. 3). The availability of this in vitro model holds 28 promise for studies into disease states, as well as probiotic discovery through bacterial factors that 29 modulate NET release, as seen by the differing amounts of NET release during different bacterial 30 cocultures.

31 Together, our observations reveal that the genomic detection provided the scaffold information by 32 which NETs release functional proteins to the extracellular milieu. PMA was sufficient to 33 produce replicable amounts of NET in a dose response manner, impacting the results of our 
1 proteomics and genomic analysis. Our NET database will be a valuable tool to identify

2 similarities and differences for gene and protein expression between primary and cell lines and

3 improve our understanding of NET biology and pathology.

5 Acknowledgments

6 General: We would like to thank the J. Craig Venter Institute Sequencing Core for their tireless 7 efforts to provide clean sequencing data and storage support. We would also like to thank 8 Timothy Meerloo, Director of the UCSD Electron Microscopy Core for assistance in sample 9 preparation and training.

10 Funding: This work was supported in part by US Public Health Service grant DE025383 from 11 the National Institutes of Dental and Craniofacial Research and by the J. Craig Venter Institute 12 given to M.F. and by the National Science Foundation grant HRD1302973 given to D.S. .

13 Author contributions: M.F. conceptualized, designed the study. D.S. performed experiments and 14 partial bioinformatics analysis. W.L. performed genomic data analysis. Y.Y., YH.L. performed 15 proteomics experiments and performed data analysis.

16 All authors wrote the manuscript and D.S and M.F edited for clarity.

17 Competing interests: The authors declare that they have no competing interests.

18 Data and materials availability: The mass spectrometry proteomics data have been deposited to 19 the ProteomeXchange Consortium via the PRIDE partner repository with the dataset identifier 20 PXD016143. The raw genomic sequences are available from NCBI Sequence Read Archive 21 (SRA) under BioProject under accession PRJNA587717. Sliding window files of aforementioned 22 sizes and unmappable read files are available through the corresponding author.

\section{$24 \quad$ References and Notes}

251 Brinkmann V, Reichard U, Goosmann C, Fauler B, Uhlemann Y, Weiss DS et al. Neutrophil 26 extracellular traps kill bacteria. Science 2004; 303: 1532-1535.

272 Zuo Y, Yalavarthi S, Shi H, Gockman K, Zuo M, Madison JA et al. Neutrophil extracellular 28 traps in COVID-19. JCI Insight 2020. doi:10.1172/jci.insight.138999. 
13 Nathan C. Neutrophils and immunity: challenges and opportunities. Nat Rev Immunol 2006;

2 6: $173-182$.

34 Urban CF, Reichard U, Brinkmann V, Zychlinsky A. Neutrophil extracellular traps capture

4 and kill Candida albicans yeast and hyphal forms. Cell Microbiol 2006; 8: 668-676.

55 Saitoh T, Komano J, Saitoh Y, Misawa T, Takahama M, Kozaki T et al. Neutrophil

extracellular traps mediate a host defense response to human immunodeficiency virus-1. Cell Host Microbe 2012; 12: 109-116.

86 Abdallah DSA, Lin C, Ball CJ, King MR, Duhamel GE, Denkers EY. Toxoplasma gondii 9 triggers release of human and mouse neutrophil extracellular traps. Infect Immun 2012; 80:

$10 \quad 768-777$.

7 Walker MJ, Hollands A, Sanderson-Smith ML, Cole JN, Kirk JK, Henningham A et al. DNase Sda1 provides selection pressure for a switch to invasive group A streptococcal infection. Nat Med 2007; 13: 981-985.

Branzk N, Lubojemska A, Hardison SE, Wang Q, Gutierrez MG, Brown GD et al. Neutrophils sense microbe size and selectively release neutrophil extracellular traps in response to large pathogens. Nature Immunology. 2014; 15: 1017-1025.

9 Urban CF, Ermert D, Schmid M, Abu-Abed U, Goosmann C, Nacken W et al. Neutrophil extracellular traps contain calprotectin, a cytosolic protein complex involved in host defense against Candida albicans. PLoS Pathog 2009; 5: e1000639.

$10 \mathrm{Yu}$ Y, Su K. Neutrophil Extracellular Traps and Systemic Lupus Erythematosus. J Clin Cell Immunol 2013; 4. doi:10.4172/2155-9899.1000139.

11 Pullan J, Greenwood H, Walton GM, Stockley RA, Sapey E. Neutrophil extracellular traps (NETs) in COPD: A potential novel mechanism for host damage in acute exacerbations. Eur Respir J 2015; 46: PA5055.

12 Wong SL, Demers M, Martinod K, Gallant M, Wang Y, Goldfine AB et al. Diabetes primes neutrophils to undergo NETosis, which impairs wound healing. Nat Med 2015; 21: 815-819.

13 Pinegin B, Vorobjeva N, Pinegin V. Neutrophil extracellular traps and their role in the development of chronic inflammation and autoimmunity. Autoimmun Rev 2015; 14: 633-640.

14 Ley K, Hoffman HM, Kubes P, Cassatella MA, Zychlinsky A, Hedrick CC et al. Neutrophils: New insights and open questions. Sci Immunol 2018; 3. doi:10.1126/sciimmunol.aat4579.

$31 \quad 15$ He Y, Yang F-Y, Sun E-W. Neutrophil Extracellular Traps in Autoimmune Diseases. Chin $32 \quad$ Med J 2018; 131: 1513-1519.

3316 Papayannopoulos V. Neutrophil extracellular traps in immunity and disease. Nat Rev Immunol 34 2018; 18: 134-147.

3517 Krishnamoorthy N, Douda DN, Brüggemann TR, Ricklefs I, Duvall MG, Abdulnour R-EE et 
118 Boeltz S, Amini P, Anders H-J, Andrade F, Bilyy R, Chatfield S et al. To NET or not to

NET:current opinions and state of the science regarding the formation of neutrophil extracellular traps. Cell Death Differ 2019; 26: 395-408.

19 Schauer C, Janko C, Munoz LE, Zhao Y, Kienhöfer D, Frey B et al. Aggregated neutrophil extracellular traps limit inflammation by degrading cytokines and chemokines. Nat Med 2014; 20: $511-517$.

20 Tarella C, Ferrero D, Gallo E, Pagliardi GL, Ruscetti FW. Induction of differentiation of HL60 cells by dimethyl sulfoxide: evidence for a stochastic model not linked to the cell division cycle. Cancer Res 1982; 42: 445-449.

21 QIAGEN. AllPrep DNA/RNA/Protein Mini Handbook - QIAGEN. https://www.qiagen.com/us/resources/resourcedetail?id=58d2f796-181a-49df-87188057e370014d\&lang=en (accessed 10 Jan2020).

22 Najmeh S, Cools-Lartigue J, Giannias B, Spicer J, Ferri LE. Simplified Human Neutrophil Extracellular Traps (NETs) Isolation and Handling. J Vis Exp 2015. doi:10.3791/52687.

23 HaileMariam M, Eguez RV, Singh H, Bekele S, Ameni G, Pieper R et al. S-Trap, an Ultrafast Sample-Preparation Approach for Shotgun Proteomics. J Proteome Res 2018; 17: 2917-2924.

24 Lin Y-H, Eguez RV, Torralba MG, Singh H, Golusinski P, Golusinski W et al. SelfAssembled STrap for Global Proteomics and Salivary Biomarker Discovery. J Proteome Res 2019; 18: 1907-1915.

25 Proprietary I. Sequencing System Guide. https://support.illumina.com/content/dam/illuminasupport/documents/documentation/system_documentation/novaseq/novaseq-6000-systemguide-1000000019358-11.pdf.

26 Miralda I, Uriarte SM, McLeish KR. Multiple Phenotypic Changes Define Neutrophil Priming. Front Cell Infect Microbiol 2017; 7: 217.

27 Hoppenbrouwers T, Autar ASA, Sultan AR, Abraham TE, van Cappellen WA, Houtsmuller $\mathrm{AB}$ et al. In vitro induction of NETosis: Comprehensive live imaging comparison and systematic review. PLoS One 2017; 12: e0176472.

28 Najmeh S, Cools-Lartigue J, Giannias B, Spicer J, Ferri LE. Simplified Human Neutrophil Extracellular Traps (NETs) Isolation and Handling. J Vis Exp 2015. doi:10.3791/52687.

29 Lim CH, Adav SS, Sze SK, Choong YK, Saravanan R, Schmidtchen A. Thrombin and Plasmin Alter the Proteome of Neutrophil Extracellular Traps. Front Immunol 2018; 9: 1554.

30 Xia C, Braunstein Z, Toomey AC, Zhong J, Rao X. S100 Proteins As an Important Regulator of Macrophage Inflammation. Front Immunol 2017; 8: 1908.

31 Tajima F. Determination of window size for analyzing DNA sequences. J Mol Evol 1991; 33: $470-473$.

32 Pieterse E, Rother N, Yanginlar C, Hilbrands LB, van der Vlag J. Neutrophils Discriminate between Lipopolysaccharides of Different Bacterial Sources and Selectively Release Neutrophil Extracellular Traps. Front Immunol 2016; 7: 484. 
133 Yousefi S, Gold JA, Andina N, Lee JJ, Kelly AM, Kozlowski E et al. Catapult-like release of mitochondrial DNA by eosinophils contributes to antibacterial defense. Nat Med 2008; 14: 949-953.

434 Amini P, Stojkov D, Felser A, Jackson CB, Courage C, Schaller A et al. Neutrophil extracellular trap formation requires OPA1-dependent glycolytic ATP production. Nat Commun 2018; 9: 2958.

35 Keshari RS, Jyoti A, Kumar S, Dubey M, Verma A, Srinag BS et al. Neutrophil extracellular traps contain mitochondrial as well as nuclear DNA and exhibit inflammatory potential. Cytometry A 2012; 81: 238-247.

36 Brinkmann V, Zychlinsky A. Neutrophil extracellular traps: is immunity the second function of chromatin? J Cell Biol 2012; 198: 773-783.

37 Brinkmann V. Neutrophil Extracellular Traps in the Second Decade. Journal of Innate Immunity. 2018; 10: 414-421.

1438 Rincón E, Rocha-Gregg BL, Collins SR. A map of gene expression in neutrophil-like cell 15 lines. BMC Genomics 2018; 19: 573.

\section{Figure Legends}

18 Figure 1. NET Production and Isolation. (a) Schematic of in vitro NET production. Cultured 19 HL-60 cell lines incubated with DMSO treatment differentiate to neutrophils (dHL60 cells). After which, PMA stimulation leads to NET production, isolation, and "omics" analysis. (b) 3D holotomographic microscopy images, digitally stained based on RI (refractive index) confirms the differentiation of HL-60 cells to neutrophils (dHL-60) after 5 days in differentiation media and successful release of NETs after 4-hour incubation in 1,000 nM PMA. (c. i-iii) Electron microscope images of isolated DNA samples from (i) HL-60 cells, (ii) dHL-60 cells i.e. HL-60 and neutrophil DNA extracted via spin column and (iii) released NET extracted via centrifugation steps shows the presence of lipid bilayers in each sample (indicated by arrowheads). (d) ROS generation during NET release process was measured by MitoSOX assay that indicated Mitochondrial Superoxide formation over 5 hours of incubation with PMA. (i) Doublet discrimination gating strategy was used to ensure accurate MitoSOX-red quantification. (ii) Representative panel of flow cytometry analysis shows the generation of ROS from neutrophils on incubation with PMA for 2 hours. Red circle denotes the changes in the ROS generating 32 neutrophil population over time. (e) DNA quantification by Agilent High-sensitive DNA chip 33 verifies the composition of extracted NET samples. Lane 1 shows an isolated NET DNA sample. 34 Lane 2 shows an isolated NET sample after incubation with DNase to digest all DNA contents. 
1 Arrows indicate the electropherogram of each sample in the gel image above. Lane L shows the 2 DNA ladder marker.

4 Figure 2. NET Proteome. (A) Proteome analysis of NET from three representative samples (i.e. 5 rep1, rep2, and rep3) identified a total of 2,364 proteins after Benzonase treatment and 1,711 6 proteins in untreated samples. Common proteins found among three representative samples in 7 Benzonase treated and untreated NET is 1,358 and 1,008, respectively. (B) Dynamic range of the 8 NET proteome. Data showing (1,722 proteins) here is from Benzonase-treated NET. Median 9 values of the three replicate experiments were used for the plot. Previously reported proteins 10 associated with NET by Urban et. al. are denoted by orange dots, most of which ranked among 11 the 100 most abundant proteins found in our NET samples. (Median value of three experimental 12 replicates are plotted here). (C) Hierarchical clustering of the 126 significant proteins (fold 13 change $\geq 2$ or $\leq-2$; Permutation FDR 0.05) between the two groups. Z-scored LFQ intensities 14 were color coded as indicated in the scale bar. (D) STRING protein network and Gene Ontology 15 analysis of 101(out of 126) significantly enriched proteins in NETs after Benzonase treatment 16 were analyzed using embedded STRING app in CytoScape software (version 3.7.2). The 17 confidence score cutoff was set to 0.4. Representative enriched gene ontology (GO) terms (e.g., 18 biological process, molecular function and cellular compartment) and corresponding FDR values 19 were depicted in the network.

Figure 3. NET DNA Enrichment/Depletion Throughout Human Genome. A 1.5-fold cutoff enrichment screen was used to determine regions of enrichment/depletion. Using annotated gene coding regions, a comparison to published expression data was used to determine overlap. The resultant circos plot for chromosomes 1-22 is shown. From innermost to outermost tracks: heatmap in order of HL-60, neutrophil (dHL-60), and NET enrichments; linkers from heatmap to color coded chromosomes in order from red (chromosome 1) clockwise to pink (chromosome 22); and linkers to gene names.

Figure 4. NET Genomic Enrichment Regions. All comparisons are made between HL-60, neutrophils (dHL-60), and NET samples. (a) A priori gene comparison based on proteomics results, previously reported proteins by Urban et al., and known biochemical pathways associated with NET formation. (p-value: *<0.05) (b) Comparison of telomere counts (TTAGGGTTAGGG) across all chromosomes for each sample (ANOVA, p < 0.05). (c) Normalized mitochondrial 
1 enrichment quantification from two sequencing runs ( $\mathrm{n}=2$ per sample, ANOVA, $\mathrm{p}<0.05)$. (d)

2 Mitochondrial enrichment by position number (sliding window size $=500 \mathrm{nts}$ ).

5 Table 1. List of Reagents and Platforms.

\begin{tabular}{|c|c|}
\hline Reagent/Item Description & Reference number \\
\hline HL-60 cell line & ATCC, cat. no. CCL-240 \\
\hline IMDM & Gibco, cat. no. 12440061 \\
\hline FBS & ATCC, cat. no. $30-2020$ \\
\hline Penicillin + Streptomycin & Gibco, cat. no. 15140122 \\
\hline Cell Imaging Cytometer & Nexcelom, CeligoS, cat. no. 200 -BFFL-S \\
\hline Agilent Bioanalyzer 2100 & Agilent, model G2939A \\
\hline QuBit 2.0 & Invitrogen, cat. no. Q32866 \\
\hline Qiagen AllPrep DNA/RNA/Protein Minikit & Qiagen, cat. no. 80004 \\
\hline Ultracentrifuge tube & cat. no. $149569 \mathrm{C}$ \\
\hline $\begin{array}{l}\text { On-Chip Sort Flow Cytometer-2D Chip- } \\
\text { Z1001 }\end{array}$ & cat. no. 1002004 \\
\hline $\begin{array}{l}\text { MitoSOXTM Red Mitochondrial Superoxide } \\
\text { Indicator }\end{array}$ & Invitrogen, cat. no. M36008, lot 2015529 \\
\hline Eosin $Y, 0.25 \%(w / v)$ in 57\% Alcohol & Ricca Chemical, cat. no. 284516 \\
\hline $\begin{array}{l}\text { Hematoxylin Stain Solution, Gill } 1 \\
\text { formulation, regular strength }\end{array}$ & Ricca Chemical, cat. no. 353516 \\
\hline Giemsa Stain Solution & LabChem, Inc., cat. no. LC148407 \\
\hline Confocal Microscope & Leica TCS SP5 II \\
\hline $\begin{array}{l}\text { Cytofix/Cytoperm Fixation } \\
\text { Permeabilization solution }\end{array}$ & BD Biosciences, cat. no. 55472251-2090KZ \\
\hline DAPI & Invitrogen, cat. no. D1306 \\
\hline Cytoseal-60 mounting media & Thermo Scientific, cat. no. 83104 \\
\hline Tomographic Microscope & Nanolive 3D Cell Explorer \\
\hline Scanning Electron Microscope & Leica UCT Ultramicrotome \\
\hline
\end{tabular}


Grid Viewing Microscope

Grid Examination

Grid Recording Camera

NexteraXT library prep kit

Sequencing Platform

Flow Cell

DNA Mapping Software

Neutrophil Elastase ELISA
Tecnai G2 Spirit BioTWIN transmission electron microscope equipped with an Eagle $4 \mathrm{k}$ HS digital camera (FEI)

100 mesh, Electron Microscopy Sciences, Hatfield, PA) uranyl acetate (Ladd Research Industries, Williston, VT

EOL JEM-1400Plus transmission electron microscope operating at $80 \mathrm{kV}$

Gatan OneView 4K

Illumina, cat. no. FC-131-1096

Illumina, NovaSeq 6000

S2 2X150bp

CLC Workbench v11, v12

Abcam, cat. no. ab204730 


\section{Figure 1}

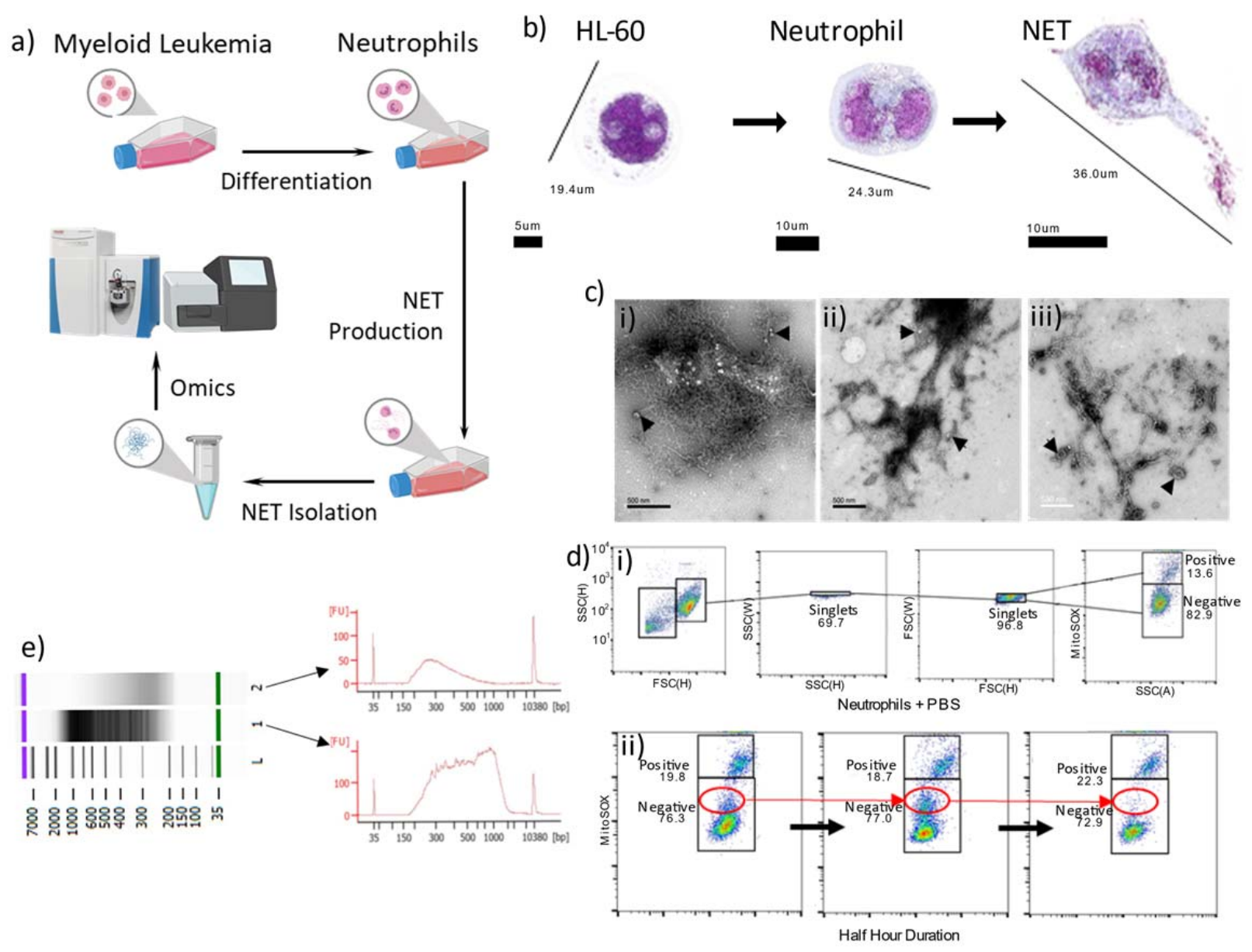


bioRxiv preprint doi: https://doi.org/10.1101/2020.05.18.102772; this version posted May 22, 2020. The copyright holder for this preprint (which was not certified by peer review) is the author/funder. All rights reserved. No reuse allowed without permission.

Figure 2

$\underline{A}$
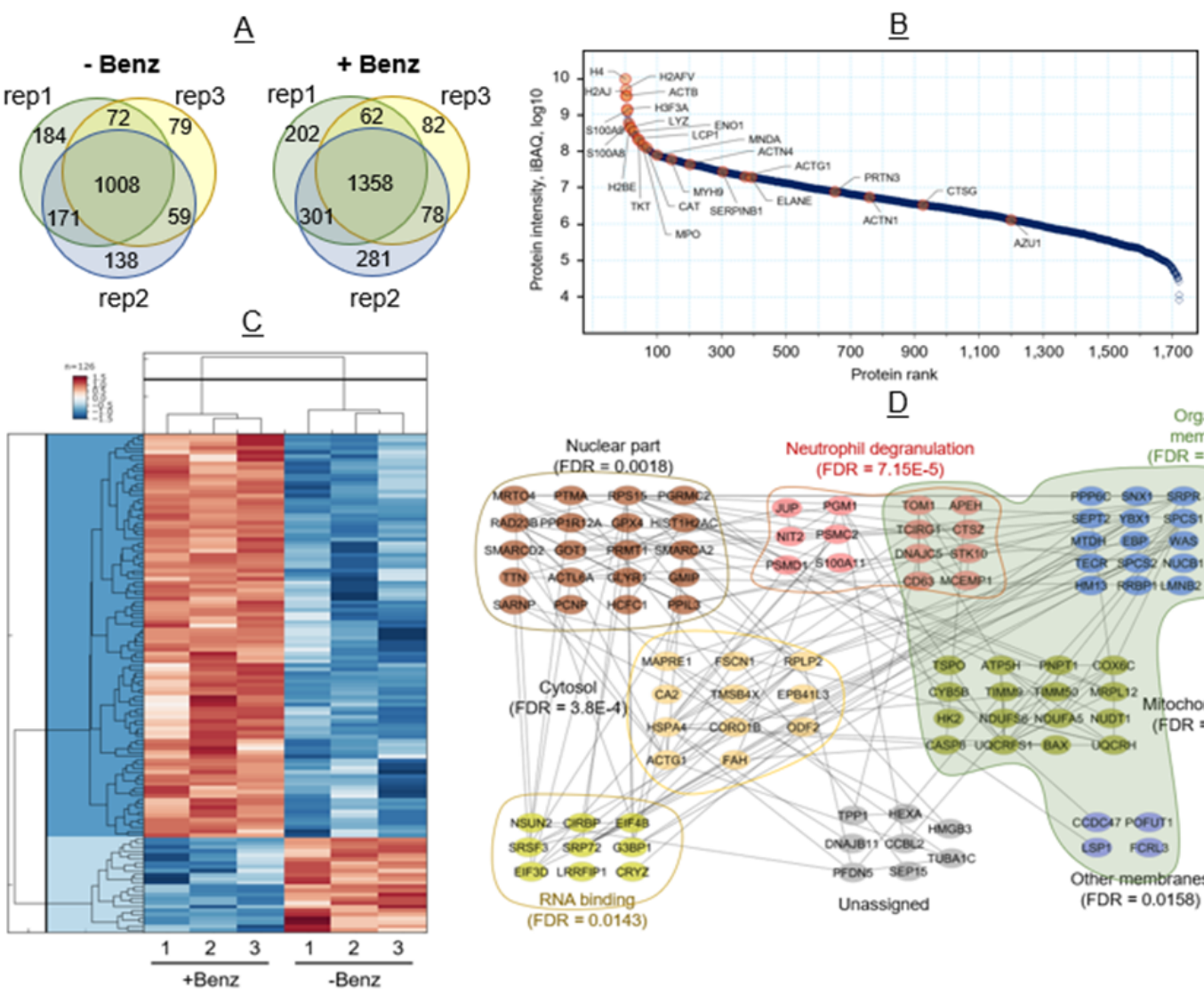

$\underline{\mathrm{D}}$

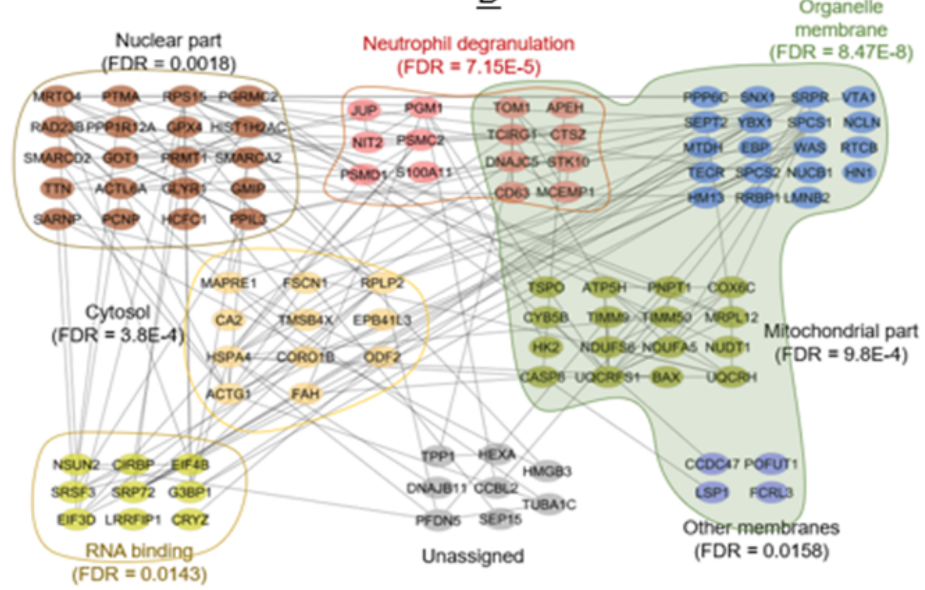


bioRxiv preprint doi: https://doi.org/10.1101/2020.05.18.102772; this version posted May 22, 2020. The copyright holder for this preprint (which was not certified by peer review) is the author/funder. All rights reserved. No reuse allowed without permission.

\section{Figure 3}

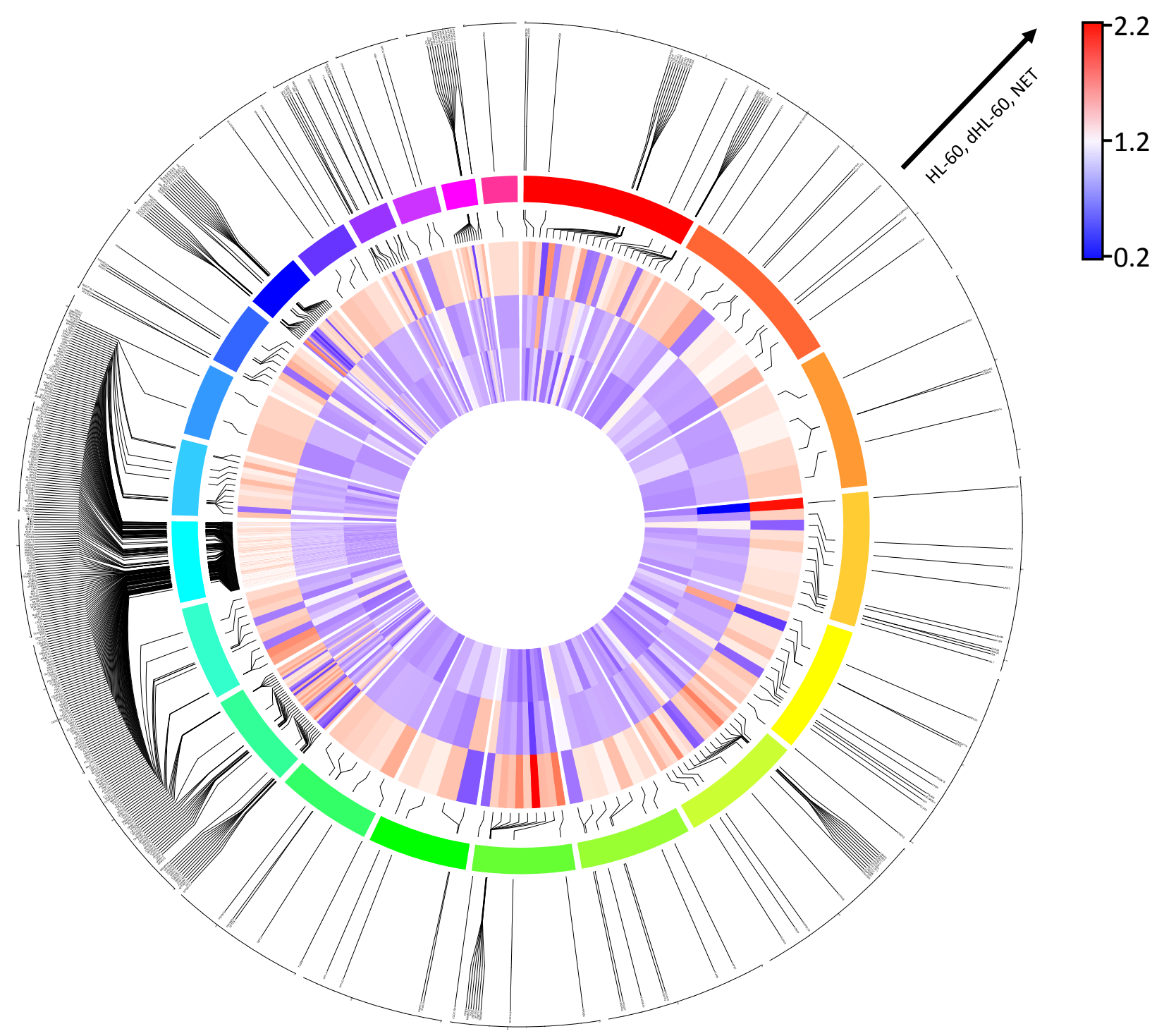




\section{Figure 4}
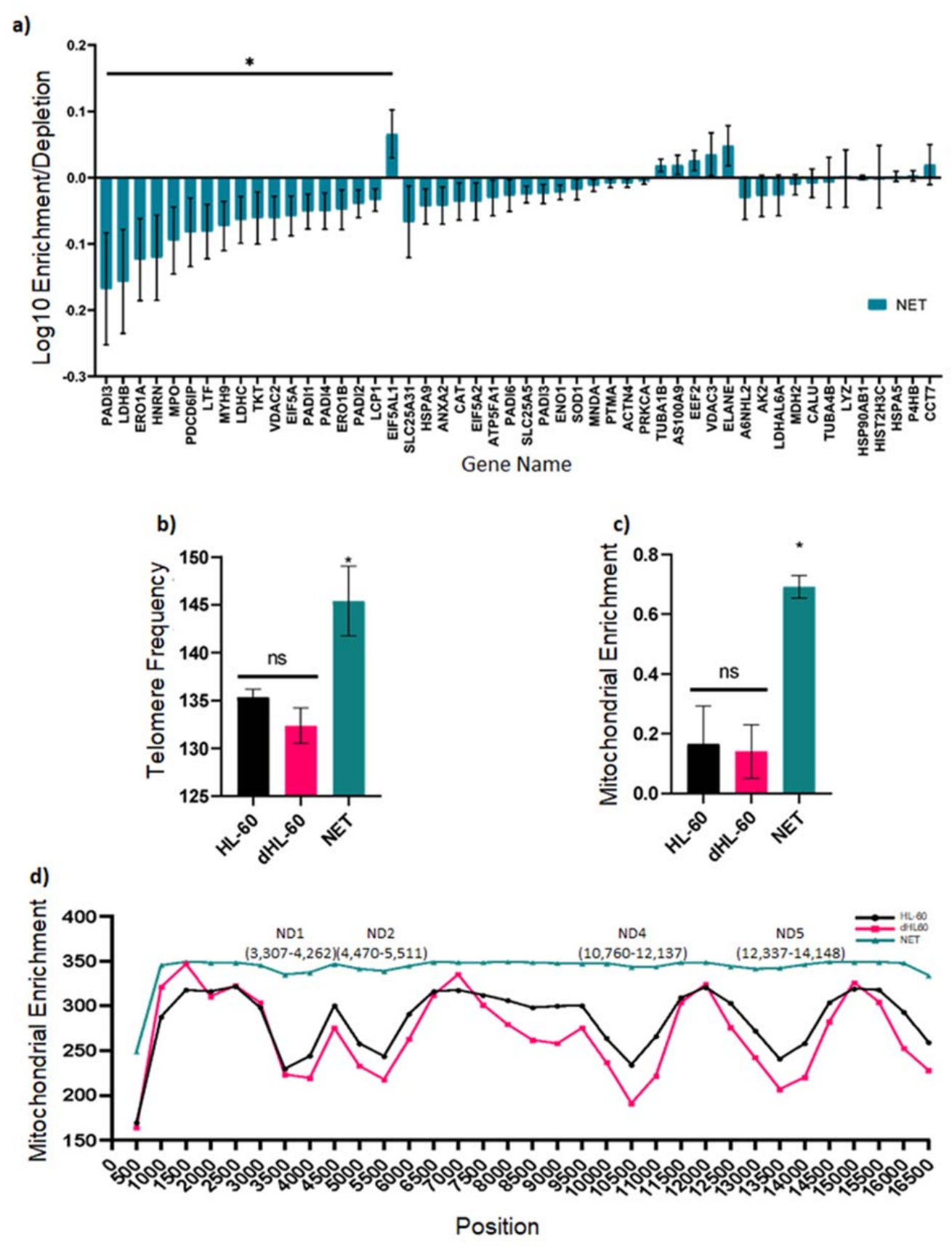